Check for updates

Cite this: J. Mater. Chem. B, 2019, 7, 2997

Received 1st February 2019, Accepted 17th March 2019

DOI: $10.1039 / c 9 t b 00238 c$

rsc.li/materials-b

\title{
Cemented injectable multi-phased porous bone grafts for the treatment of femoral head necrosis $f$
}

\author{
Wei Zhu, ${ }^{a}$ Qi Ma, ${ }^{a}$ Sebastian Borg, ${ }^{b}$ Caroline Öhman Mägi, ${ }^{b}$ Xisheng Weng, ${ }^{* a}$ \\ Håkan Engqvist ${ }^{b}$ and Wei Xia (D) *b
}

\begin{abstract}
Femoral head necrosis (FHN) can induce musculoskeletal disability. It presents a challenge from diagnostic and therapeutic points of view. Open surgery for the treatment of FHN is not an optimal route. To minimize the surgery window, an injectable material with a porous structure and bioactive nature is preferred. The fabrication of an injectable porous bone graft via a simple route was the aim of our study. Therefore, cemented multi-phased calcium phosphate porous granules have been studied with varied phase compositions, pore sizes and porosities, and degradation rates. Granules templated by PEG 100-600 $\mu \mathrm{m}$ were chosen for cell toxicity and in vitro osteogenic potential testing. Rabbits, making up a femoral head necrosis model, were implanted with granule A. Mature cancellous bone tissue was observed in the femoral head defect after 2 months implantation. The results indicate that the newly formed injectable bioactive porous grafts could be a good candidate for the treatment of femoral head necrosis.
\end{abstract}

\section{Introduction}

Femoral head necrosis (FHN) is an increasingly common cause of musculoskeletal disability as well as a major diagnostic and therapeutic challenge. ${ }^{1-3}$ Diagnosis can monitor the progress of the disease and degeneration of the femoral head over time, which ultimately leads to failure of the joint. FHN can lead to the collapse of the femoral head and painful arthritis of the hip joint. Although the disease usually occurs roughly between 30 to 50 years of age and is relatively common, the underlying reasons why it occurs are not fully clear. ${ }^{4}$ It is possible to give the patient early treatment if the disease is discovered in an initial stage, which can prevent the destruction of the femoral head, which would then require surgery where the whole joint is replaced. Early treatment could drastically decrease the suffering of the patient and make a hip replacement avoidable.

At present, protected weight-bearing, core decompression, osteotomy, and vascularised or nonvascularised bone grafting have been studied to deal with osteonecrosis, but the results are far from expectations. ${ }^{5}$ There is an extreme need in clinics.

\footnotetext{
${ }^{a}$ Department of Orthopedics, Peking Union Medical College Hospital, Chinese Academy of Medical Sciences \& Peking Union Medical College, Beijing 100730, China. E-mail: xshweng@medmail.com.cn; Fax: +86 1069152820; Tel: +8613366200018

${ }^{b}$ Applied Materials Science, Department of Engineering Science, Uppsala University, Uppsala 75121, Sweden. E-mail: wei.xia@angstrom.uu.se; Fax: +46 184713572; Tel: +46184717961

† Electronic supplementary information (ESI) available. See DOI: 10.1039/ c9tb00238c
}

During the last few decades, different ceramic materials have proved to be a very promising group of materials to replace bone autografts and allografts. ${ }^{6-8}$ They eliminate the risk of disease transfer, they can be stored for a long time, they are made of abundant raw materials and they are osteoconductive. They can also be used for injuries of all sizes since they can be custom made.

The by far most common group of ceramic materials is calcium phosphates (CaPs). ${ }^{9-13}$ Important compounds in this group of materials are HA, alpha/beta-tricalcium phosphate $(\alpha / \beta$-TCP) and brushite. $\alpha$-TCP and $\beta$-TCP have been used in different clinical applications, such as dentistry, maxillofacial repair and orthopaedics. ${ }^{8,11,14-16} \alpha$-TCP is the major component of many hydraulic bone cements. $\beta$-TCP is mainly used as bioactive and biodegradable ceramics and one of the main powder components in brushite cements. Generally, the solubility of $\alpha$-TCP is higher than $\beta$-TCP. $\beta$-TCP has showed good results in different studies and resolves more rapidly than HA. At the same time, its osteoconductive properties lead to the formation of new bone. The new bone then takes the place of the $\beta$-TCP implant, as in the case with bone auto- or allografts. ${ }^{17-19}$ Although the mineral part of the human bone is mainly made up of HA, it has been used as a "gold" orthopaedic implant material for a long time. Synthetic $\beta$-TCP generally contains calcium pyrophosphate (CPP), which has been proved to have an enhanced effect on stimulating new bone formation. ${ }^{20}$ Therefore, calcium phosphate-based materials have shown good compatibility, bioactivity, and osteoconduction in bone repair and regeneration. The form of porous CaP granules/ scaffolds is a naturally candidate for the treatment of FHN. 
Bioactive ceramic materials are the most promising synthetic bone graft in the market. Finding a method to make a better bone graft is the dream of all biomaterials scientists. A ceramic graft can be produced in several ways. Different casting methods and sintering are common. However, bone grafts can also be produced by an acid-base reaction where a solid phase is mixed with a liquid phase, resulting in a selfsetting material. Apart from the stoichiometry and the crystal structure of the material, the morphology and microstructure are also very important for the success of the implant. The in vitro and in vivo properties are influenced by the size, shape, porosity (including pore size) and surface structure of the implant. ${ }^{11,21-23}$ The microstructure of porous calcium phosphate ceramics could not only improve the osteoconduction but also induce osteointegration. ${ }^{24,25}$ It is possible to make a solid implant, which is made to fit the injury, but the use of granules would provide a much larger surface area since the cells can then grow in between the individual granules.

Several studies have concluded that the critical pore size for new bone formation is around $100 \mu \mathrm{m} .^{22,23,26,27}$ If the pores are smaller, they are penetrated by osteoid mineral and fibrous tissue. In order to get vascularization, the formation of blood vessels, pores with a size of several hundred micrometers are required. A porous graft is also well suited for use as a matrix for an activated implant. An activated implant could, for example, be loaded with stem cells, which are hypothesized to provide osteogenic properties.

Injectable bone grafts could enable a minimized operation to help patients suffer less pain. A synthetic multi-phased calcium phosphate, such as HA/ $\alpha / \beta-\mathrm{TCP} / \mathrm{CPP}$, could combine the advantages of the components for bone repair. ${ }^{28-32}$ Especially for CPP, Palmer et al., reported that CPP could have the potential effect of osteoinduction under a certain content. ${ }^{20}$ Introducing a small amount of CPP into a multi-phased bioceramic graft could enhance its osteogenic properties. The conventional ways of producing a porous structure in ceramics always involve high-temperature calcination. In this study, we developed an environmentally friendly and reproducible process for producing porous bone granules without using extra instrumentation. The pore size and composition were well controlled. The best formulation was chosen for in vitro and in vivo testing.

\section{Materials and methods}

\subsection{Preparation of multi-phase porous granules}

Porous granules where made by mixing $1.94 \mathrm{~g}$ of $\alpha$-TCP (RMS Foundation, Switzerland), $0.060 \mathrm{~g}$ of $\beta$-TCP (Sigma Aldrich, Germany) and $3.0 \mathrm{~g}$ of $20000 \mathrm{~g} \mathrm{~mol}^{-1}$ poly(ethylene glycol) (PEG, Sigma Aldrich, Germany). Before weighing the PEG, it was melted at $70{ }^{\circ} \mathrm{C}$ on aluminium foil for one to two hours. After melting, the PEG was allowed to cool and solidify at room temperature, after which it was moulded using a mortar and sieved to 100-600 $\mu \mathrm{m}$. PEG with size fractions of 100-200 $\mu \mathrm{m}$ and 200-400 $\mu \mathrm{m}$ was also prepared with the same method.
The powder phase was mixed for five minutes using a CapVibrator (Ivoclar Vivadent, USA). A liquid phase consisting of a $2.5 \%$ $\mathrm{Na}_{2} \mathrm{HPO}_{4}$ (S9763, Sigma Aldrich, Germany) solution was prepared using a volumetric flask.

$0.8 \mathrm{ml}$ of the liquid phase was added to the powder phase using a pipette (Pipet-Lite LTS Pipette L-1000XLS+, Mettler Toledo, Switzerland). The cement was mixed for one minute using a CapVibrator (Ivoclar Vivadent, USA). After mixing, the cement was moulded in Teflon moulds with a diameter of $1.2 \mathrm{~mm}$ and a height of $1.2 \mathrm{~mm}$. The moulds with the cement were covered and left to set at room temperature for 48 hours. After this, the granules were de-moulded and put in a glass beaker. 300-400 $\mathrm{ml}$ of water was added and the granules were swirled around while the water was changed a couple of times. Afterwards, the granules were stored at $70{ }^{\circ} \mathrm{C}$ with water for around four hours to leach them of the PEG. The water was changed each hour. After leaching, the water was removed and the granules were stored again at $70{ }^{\circ} \mathrm{C}$ for 48 hours, for drying. Table 1 shows three types of injectable porous granules with different pore size and distribution. Finally, one chosen group was tested in vitro and in vivo based on the properties of the granules.

\subsection{Characterization methods}

$\mathrm{X}$-ray diffraction (XRD) analysis of the granules after leaching, drying and autoclaving was conducted on a Siemens Diffractometer 5000 with $\mathrm{Cu}(\mathrm{K} \alpha)$ radiation under the operating conditions of $40 \mathrm{kV}$ and $40 \mathrm{~mA}$. Crystal phase identification was determined by the database from the ICDD (International Centre for Diffraction Data). The phase composition was calculated based on XRD data using Profex software (Profex, Nicola Döbelin, Switzerland).

The morphology and 3D tomography of the samples were analysed by using scanning electron microscopy (SEM) (TM-1000, Hitachi, Ltd, Japan) and $\mu$ CT (Skyscan 1172, Bruker Corporation, Germany). The porosity of the granules ( $n=3$ ) was determined using micro-computed tomography. Reconstruction of the cross sections was done using the software package NRecon (SkyScan, Bruker, Kontich, Belgium). The reconstructed images were binarized using an optimized global threshold and structural analyses were done in the software package CTAn (SkyScan, Bruker, Kontich, Belgium). Three-dimensional reconstructions of the samples were obtained using CTvox (SkyScan, Bruker, Kontich, Belgium).

The granule degradation experiment was conducted in a phosphate buffer solution $(\mathrm{pH}=7.4)$. Groups of 10 granules were immersed in $10 \mathrm{ml}$ solutions for 1, 3, 7 and 14 days. The weight loss of the granules was evaluated by weighing collected granules after soaking for certain times. The granules were dried at $110{ }^{\circ} \mathrm{C}$ overnight before weighing.

Table 1 Groups of injectable porous granules

\begin{tabular}{lll}
\hline Group & PEG size $(\mu \mathrm{m})$ & Number of batches \\
\hline A & $100-600$ & 3 \\
B & $100-200$ & 3 \\
C & $200-400$ & 3
\end{tabular}




\subsection{Cell proliferation and cytotoxicity test}

Preosteoblasts (MC3T3-E1, Osteoblast cell line, bought from Chinese Academy of Medical Sciences) were cultured in an alpha-minimum essential medium ( $\alpha$-MEM) supplemented with $5 \%$ fetal bovine serum, $2 \mathrm{~mm} \mathrm{~L}^{-1}$ glutamine, and $100 \mu \mathrm{g} \mathrm{ml} \mathrm{m}^{-1}$ penicillin-streptomycin and incubated at $37{ }^{\circ} \mathrm{C}$ in a humidified atmosphere with $5 \% \mathrm{CO}_{2} .10 \mathrm{ml}$ of a-MEM and $500 \mathrm{mg}$ of biomaterials were mixed and placed in an incubator at $37{ }^{\circ} \mathrm{C}$ for $24 \mathrm{~h}$. The medium was collected and filtered with a $0.22 \mu \mathrm{m}$ mesh for cell culture. $2000 \mathrm{MC} 3 \mathrm{~T} 3$ cells were inoculated in each hole of a 96-hole plate, and then $200 \mu \mathrm{l}$ of medium was added to each hole. The cells were divided into the experimental group and control group. The experimental group was added daily to the above collected medium and the normal group was added to the normal medium every day. In the first three days, $10 \mu \mathrm{l}$ of CCK-8 medium was added to each cell hole for $2 \mathrm{~h}$ and then the cells were collected. At last, the OD at $450 \mathrm{~nm}$ was measured to predict growing tendency.

\subsection{In vitro osteogenic potential of multi-phase bone grafts}

Human bone marrow mesenchymal cells (Cyagen Biosciences Company, Guangzhou, China) were passaged to the third generation and prepared to 2000 cells per $1 \mathrm{ml}$ of medium. $2 \mathrm{ml}$ of cell medium was added into every hole of a 6-well plate. Then, the cells were cultured at $37{ }^{\circ} \mathrm{C}$ in a humidified atmosphere with $5 \% \mathrm{CO}_{2}$. The cell medium was replaced every three days. The cells were divided into the control group, which was cultured by normal medium, and experimental group, which was cultured by the medium mixed with bone grafts for 24 hours. After cultivation for 14 days in the two groups, the medium was removed from the holes and the cells were washed with phosphate buffer solution twice. 4\% paraformaldehyde was added into these holes for $10 \mathrm{~min}$ followed by three cycles of distilled water washing. When the holes were dry, Alizarin Red S dye solution was dropped into each hole to cover the bottom of the plate completely for $30 \mathrm{~min}$. Pictures were taken under a microscope of these holes. Using the same method to obtain these cells in the two groups after cultivating for 14 days, ALP stationary liquid was added into these holes for $3 \mathrm{~min}$ followed by PBS washing twice. Then, ALP incubation solution was mixed into these holes avoiding light. Pictures were also taken under a microscope of these holes when they were dry.

\subsection{In vivo study: rabbit femoral head defect and osteonecrosis model}

The animal experiment was performed according to the guidelines of the National Institutes of Health. The animal experiments in this study were approved by the Ethical Inspection Committee of Peking Union Medical College Hospital (XHDW2015-0034).

2.5.1 The osteonecrosis animal model. The rabbit femoral head defect and necrosis model, published previously, ${ }^{1}$ was used in this biomaterial application study. 3-3.5 $\mathrm{kg}$ New Zealand white rabbits were chosen and kept for at least one week. $3 \%$ phenobarbital $\left(1 \mathrm{ml} \mathrm{kg}^{-1}\right)$ was injected into the auricular vein until the corneal reflex was weakened. Then, the surface hair on the left thigh of these rabbits was shaved. The area was then disinfected with iodide. The surface of the skin was cut and the muscles and fascia were separated to reveal the greater trochanter of the femur. A $2.5 \mathrm{~mm}$ diameter Kirschner needle was used to drill a 45-degree angle hole at the $2 \mathrm{~mm}$ point under the greater trochanter of the femur. The area was then frozen using liquid nitrogen for 5 seconds with a fine needle at the hole.

2.5.2 Biomaterial implantation. The perforated granules were placed inside a syringe with a diameter of $1.5 \mathrm{~mm}$ and length of $60 \mathrm{~mm}$ in advance. The syringe loaded grafts were then inserted into the femoral head defect of the New Zealand white rabbit and the granules were pushed into the defect area. Each New Zealand white rabbit was implanted with up to 100 granules until the cavity was filled. In total, 16 rabbits were used: 4 rabbits for the control group at 4 weeks, 4 rabbits for the control group at 8 weeks, 4 rabbits for the testing group at
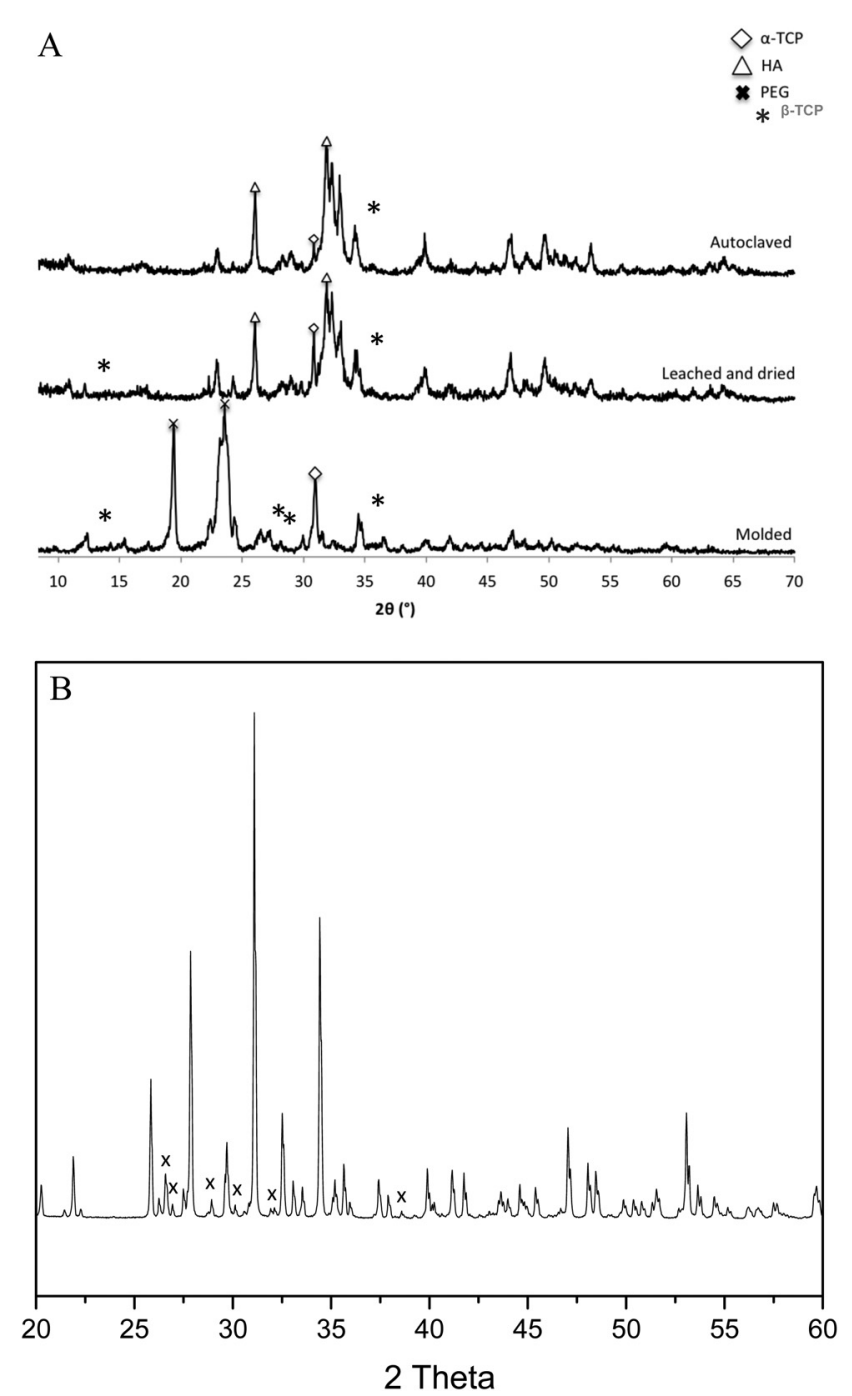

Fig. 1 (A) XRD patterns of granules after moulding (bottom), after leaching (middle) and after leaching and autoclaving (top). Peaks from $\alpha$-TCP, HA and PEG are marked. (B) XRD pattern of $\beta$-TCP, $x$ : $\beta$-calcium pyrophosphate. 


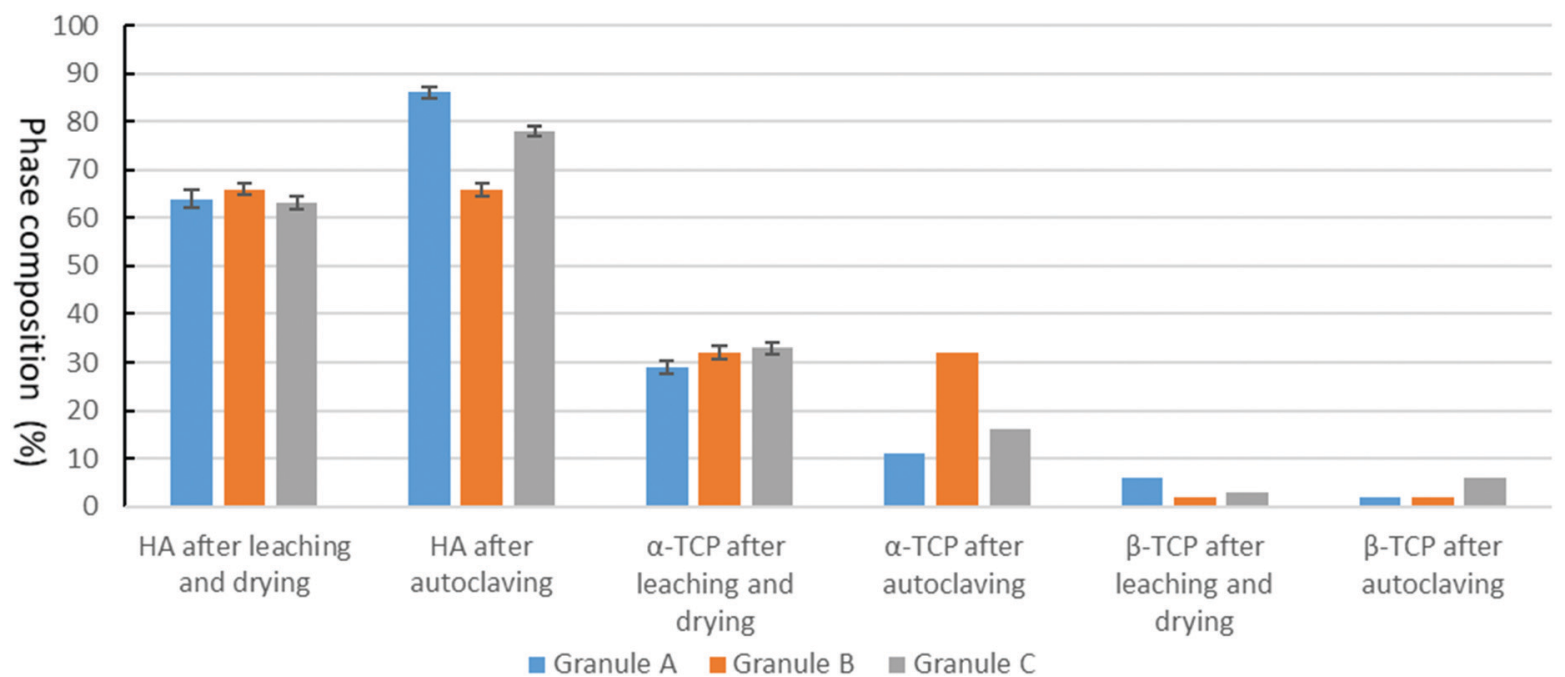

Fig. 2 Phase compositions of granules A, B and C (mass ratio, \%).

4 weeks, and 4 rabbits for the testing group at 8 weeks. Surface muscles and fascia were sutured to close the cavity. Antibiotics (penicillin $300000 \mathrm{U}$ per day) were administered every day for three days after surgery.

2.5.3 Micro-CT and histological analyses. The left femur of these New Zealand white rabbits was taken out 4 weeks and 8 weeks after implantation. Then, the femur samples were dipped in $4 \%$ paraformaldehyde. Subsequently, the samples were scanned with a micro-CT instrument (SIEMENS INVEON, $10.3 \mu \mathrm{m}$ resolution, voltage of $70 \mathrm{kV}$, and current of $400 \mathrm{~mA}$ ). Inveon Research Workplace software was used for 3D construction. The regenerated bone volume was calculated using this software with the threshold of Hu value from 400 to 1200. After the micro-CT scanning, samples were embedded in paraffin. They were then sliced into sections with a thickness of $50 \mu \mathrm{m}$ by a rotary microtome (RM2255, Leica, Germany). Then, the sections were analysed using hematoxylin and eosin (H\&E) and Sirius red staining.

2.5.4 Statistical analysis. For cell studies, CCK-8 data were expressed as mean \pm standard deviation (SD). Bone volume was expressed as mean \pm standard deviation (SD). The $T$-test was used to assess statistical significance. Probability values are expressed as follows: ${ }^{* *} P<0.001 ;{ }^{*} P<0.01 ;{ }^{*} P<0.05$.

\section{Results and discussion}

\subsection{Preparation and characterization of synthetic injectable multi-phase bone grafts}

Granule A (Fig. 1A) before leaching and drying contains $\alpha$-TCP (95\%), $\beta$-TCP (2\%) and HA (3\%). Granules B and C show similar results (Fig. S2 and S3, ESI $\dagger$ ). The raw $\beta$-TCP powder contained $10 \mathrm{wt} \%$ CPP (Fig. 1B), but no clear CPP peaks could be observed because the percentage of CPP is below $1 \%$ in the grafts. The $\alpha$-TCP peak at $30.8^{\circ}$ is clearly visible in the XRD patterns of the moulded granules but it is much smaller for the leached and autoclaved granules. Instead, HA peaks are visible for the latter at $31.8^{\circ}$. PEG has two large peaks at $19^{\circ}$ and $24^{\circ}$, which are prominent in the patterns of the moulded granules but not in those of the treated ones. $\alpha$-TCP reacted with water immediately, but slowly. Only approximately $3 \%$ of HA formed after 48 hours in air. The starting $\alpha-\mathrm{TCP} / \mathrm{H}_{2} \mathrm{O}$ ratio (in mole) was 0.13 , which is lower than the theoretical number (0.6). The lack of water would inhibit the formation of HA during the mixing and moulding.

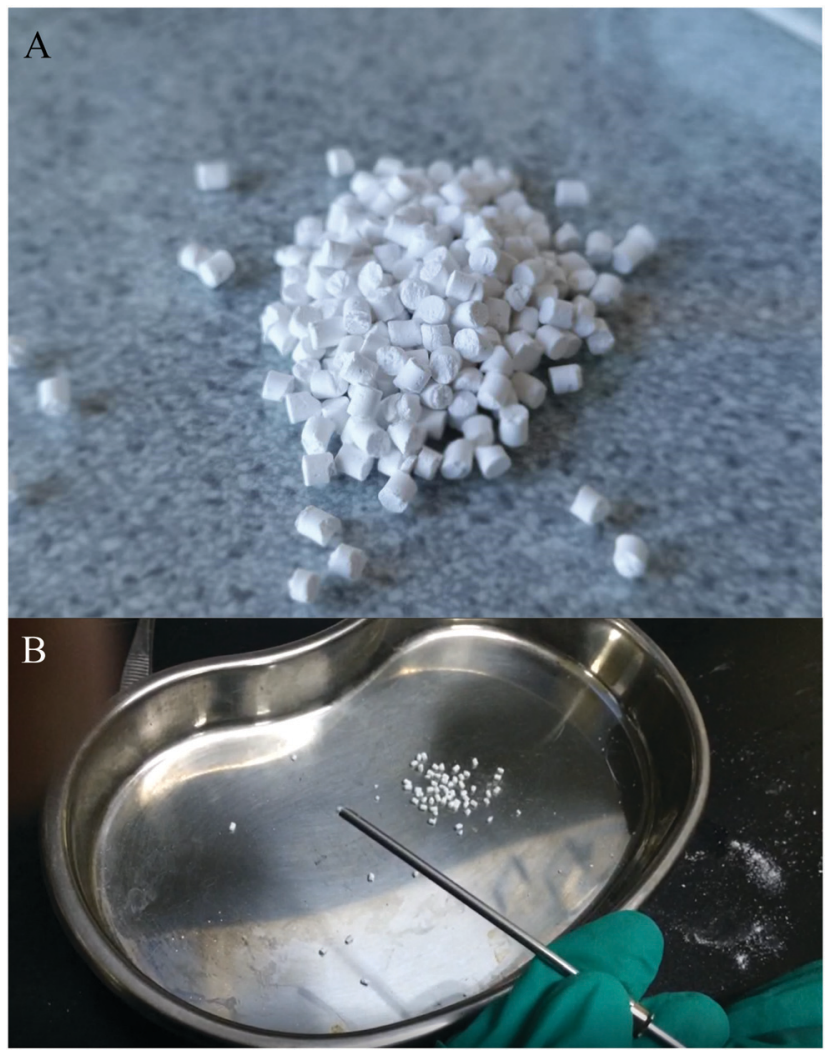

Fig. 3 (A) Optical image of granule A. (B) Granules were injected through a needle that is mainly used for bone cement. 
The leaching has two roles in the preparation of the granules: (1) to dissolve PEG to form a porous structure and (2) to form more HA phase in the granules. Fig. 2 indicates that the percentage of $\mathrm{HA}$ in all granules increased from $3 \%$ up to 64 , 66 and $63 \%$ for granules A, B and C, respectively. The percentage of $\alpha$-TCP decreased from $95 \%$ to 29,32 and 33 for granules A, B and $\mathrm{C}$, respectively. The percentage of $\beta$-TCP did not change significantly and varied from $2 \%$ to 6,3 and $2 \%$ for granules A, B and $\mathrm{C}$, respectively. Extra water and increased temperature did help the hydrolyzation of $\alpha$-TCP and the formation of the new phase, HA. During this process, $\beta$-TCP was actively involved in HA formation, therefore its relative amount did not change. Interestingly, we did not see the expected transformation of $\alpha$-TCP in all granules after autoclaving. Yes, the HA amount increased in granules $\mathrm{A}$ and $\mathrm{C}$, to 86 and 78\%, respectively, but no changes were observed for granule $\mathrm{B}$. The transformation of $\alpha$-TCP was not completed after leaching, drying and autoclaving. Therefore, a multi-phase calcium phosphate porous granule composed of HA, $\alpha$-TCP and $\beta$-TCP was prepared. Granule A contains the maximum HA phase in the final formulation.

Fig. 3 shows the optical image of the synthetic granule, and the injection of the granules. SEM analyses indicated the morphologies and pore size of all granules (Fig. 4). The size of all granules is approximately $1.2 \mathrm{~mm}$ in diameter and length, which is similar to the size of the mould. They are still rod-like, but not so perfect because the materials are brittle and part of the granules was broken after the materials were removed from the moulds. All granules are porous. Granule A has the largest pore size, except for some small pores, compared to granules B and C. Granule B shows the smallest pore size generally, and the pore size of granule $\mathrm{C}$ is in the middle. The $3 \mathrm{D}$ reconstruction images (Fig. 5) show that all granules are porous and granule B has the smallest pore size. Granule A has a broad size range. A more detailed pore size distribution is shown in Fig. 6 and 7. The porosity of granules $\mathrm{A}, \mathrm{B}$ and $\mathrm{C}$ was found to be $35.9 \pm 1.1 \%, 41.5 \pm 4.5 \%$ and $29.9 \pm 0.8 \%$, respectively. The average pore size of granules A, B and C was $119.9 \pm 17.7 \mu \mathrm{m}$, $78.3 \pm 2.4 \mu \mathrm{m}$ and $98.5 \pm 8.7 \mu \mathrm{m}$, respectively. For granule A, the range of the pore size mid-values was found to be $16-343 \mu \mathrm{m}$. The highest percentage of pores have a size of $114 \mu \mathrm{m}$ and it is $11.9 \%(\sigma=2.50 \%)$. For granule B, the range of the pore size midvalues was found to be $16-158 \mu \mathrm{m}$. The highest percentage of pores have a size of $63 \mu \mathrm{m}$ and it is $23.2 \%(\sigma=3.14 \%)$. The pore size distribution in granule B is narrower than granule A. More than $70 \%$ of the pores are in the range of $63-95 \mu \mathrm{m}$. For granule $\mathrm{C}$, the range of the pore size mid-values was found to
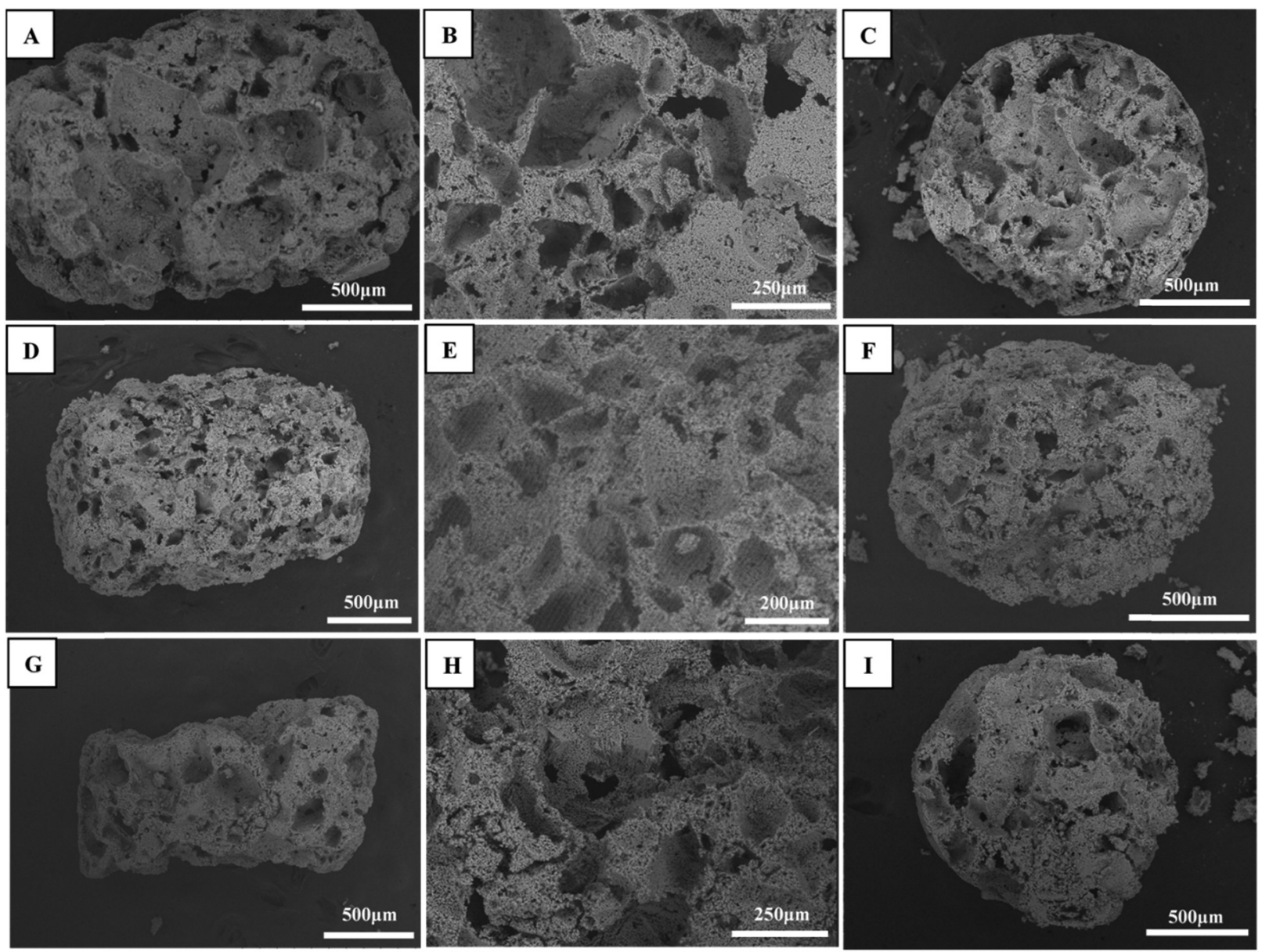

Fig. 4 Morphologies of granule A: (A) overview, (B) pore morphology, and (C) cross-section; granule B: (D) overview, (E) pore morphology, and (F) crosssection; and granule C: (G) overview, (H) pore morphology, and (I) cross-section. 
be 16-236 $\mu \mathrm{m}$. The highest percentage of pores have a size of $110 \mu \mathrm{m}$ and it is $11.1 \%(\sigma=2 \%)$. The range of pore sizes is narrower than for granule $\mathrm{A}$ but wider than for granule $\mathrm{B}$.
All obtained pore sizes were smaller than the original PEG size since the PEG sizes in granules A, B and C were 100-600, 100-200 and $200-400 \mu \mathrm{m}$, respectively. The degradation testing in the
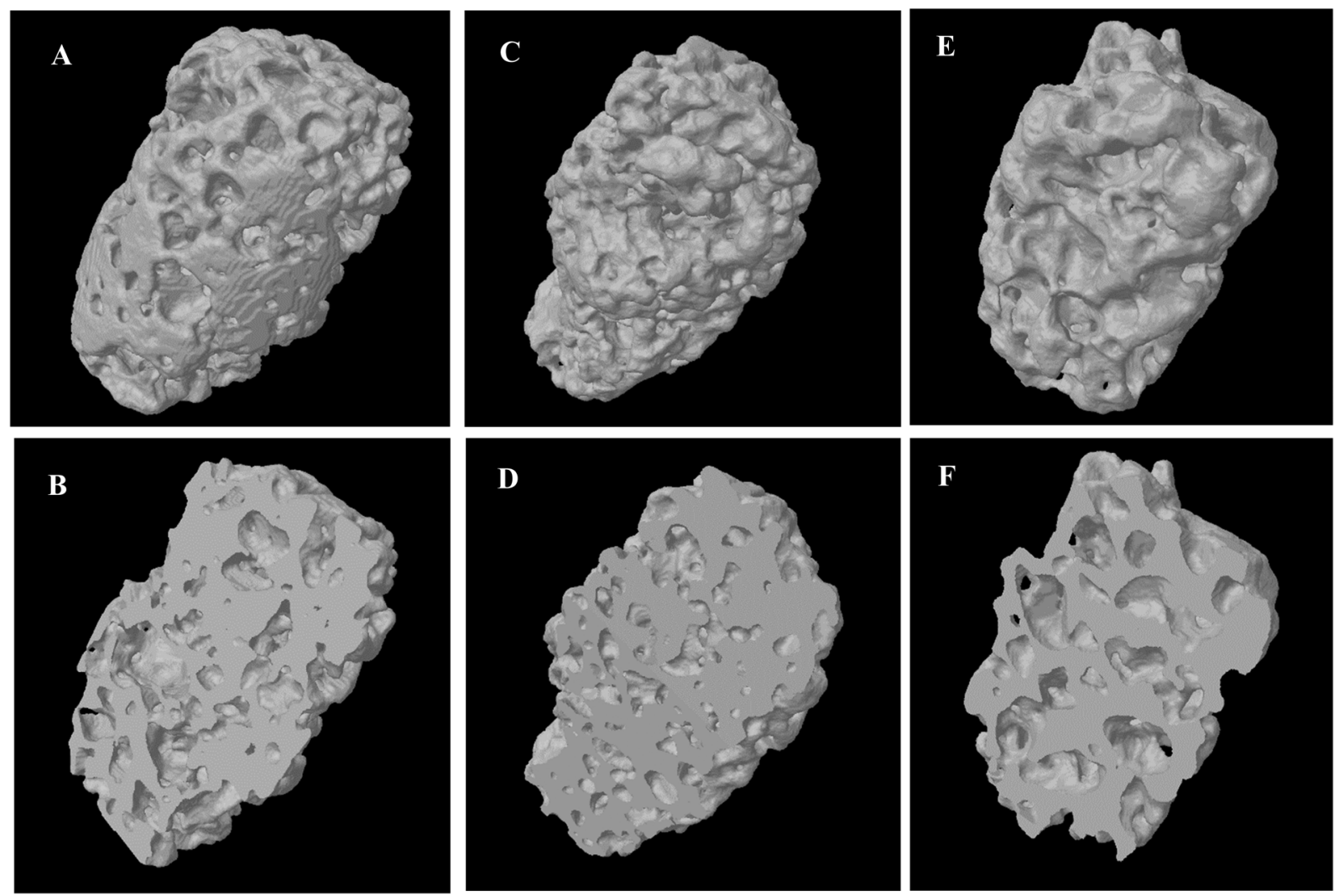

Fig. 5 Micro-CT 3D reconstruction of granule A: (A) overview and (B) cross-section; granule B: (C) overview and (D) cross-section; and granule C: (E) overview and (F) cross-section.

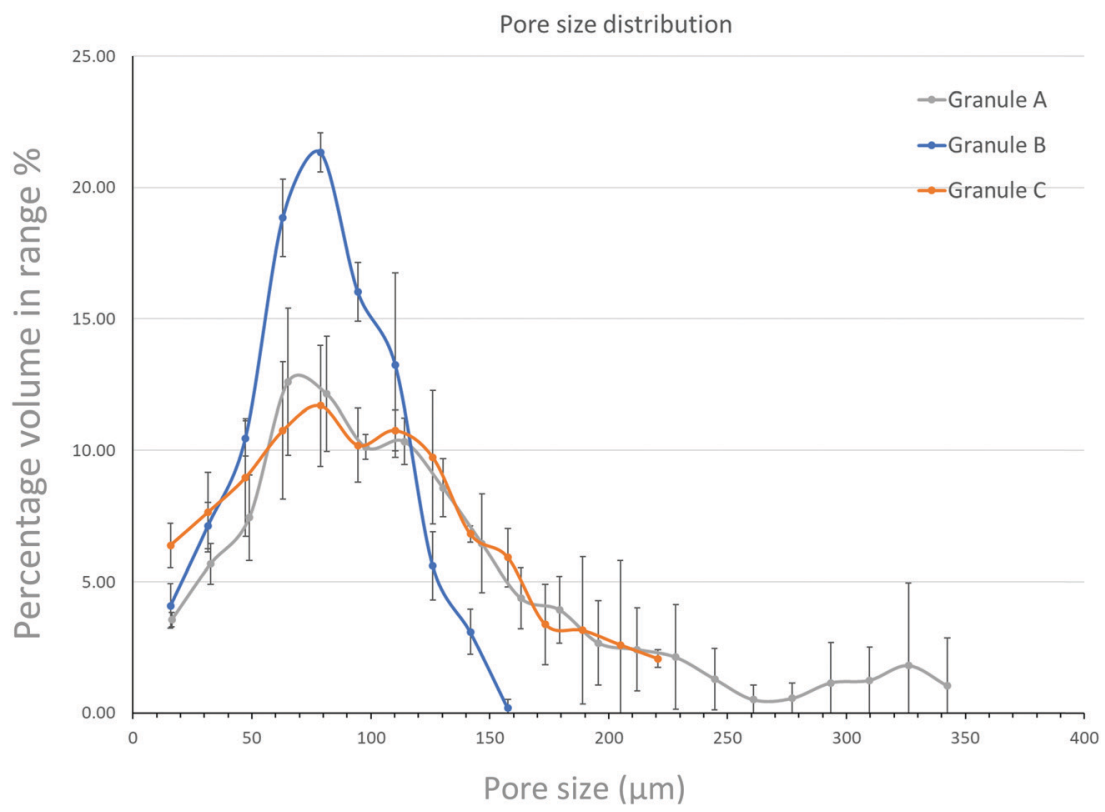

Fig. 6 Pore size distribution obtained from micro-CT (pore size below $5 \mu \mathrm{m}$ was not counted): granule A, granule B, and granule C. The profile shows the percentage of the volume in a specific pore-size range ( $x$ axis) in the total volume of the full pore-size range. 


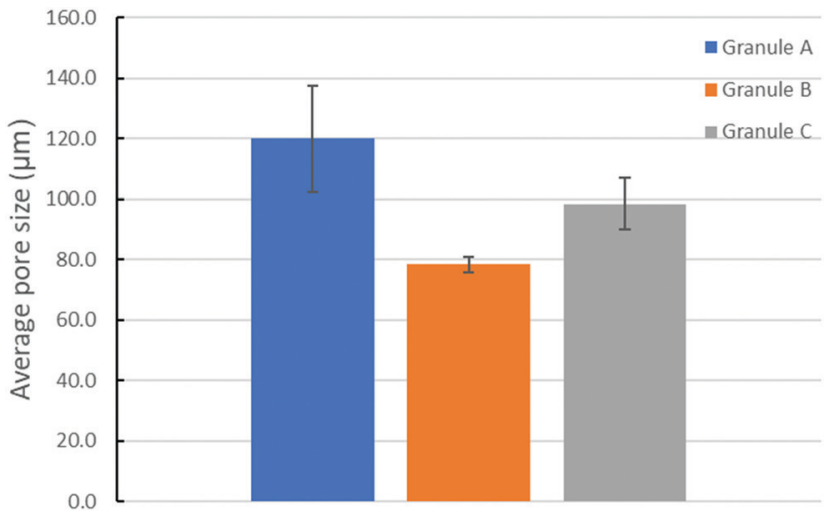

Fig. 7 The average pore size (calculated based on the results shown in Fig. 6) in granules A, B and C after drying and autoclaving. Porosity: granule A $(35.9 \pm 1.1 \%)$, granule B $(41.5 \pm 4.5 \%)$, and granule $C(29.9 \pm 0.8 \%)$.

phosphate buffer solution indicated that the weight loss of all granules increased gradually in the 14 days. Granule B degraded a bit slower, approximately $9 \%$ on day 14 . Granules A and C undergo weight loss of approximately 11 and $12 \%$ on day 14 , respectively (Fig. 8).

\subsection{Cytotoxicity}

Pre-osteoblasts (MC3T3-E1) were used to evaluate the cytotoxicity because they are an osteoblast precursor cell line derived from Mus musculus (mouse) calvaria. ${ }^{33,34}$ The cells in the experimental group were cultivated using the cell medium incubated at the bone graft without diluting. The cells in the control group were cultivated on the blank tissue culture plate. Cell Counting Kit-8 test was used to calculate the cell number in the two groups after 1, 3 and 5 days cultivation. As shown in Fig. 9, the OD values in the two groups increased in 5 days. The proliferation of cells in the experimental group increased more slowly compared with that in the control group. This shows that the experimental group has a small amount of toxicity compared to the control group, but it did not inhibit the cell proliferation. Calcium

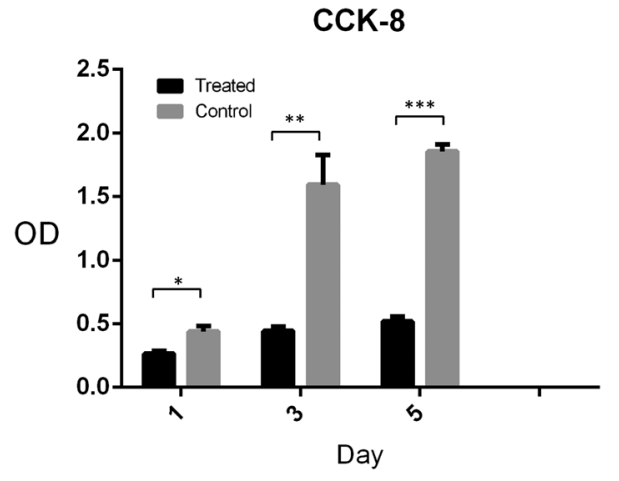

Fig. 9 Cell (MC3T3-E1) toxicity test of the cell culture in the absence and presence of the dissolution extracts of granule $A$ after culturing for one day ( ${ }^{\star} P<0.05 ;{ }^{*} P<0.01 ;{ }^{*} * P<0.001 ;$ two-tailed $T$-test).

phosphate cement has shown better biocompatibility and is a widely used biomaterial due to its chemical and structural similarity to the inorganic component of bone. ${ }^{35,36}$ The extraction medium, which triggered ion release in our study, was not diluted in the cell culture compared with other studies ${ }^{37,38}$ so that a small amount of toxicity was observed. However, it showed better performance when implanted in vivo because of the flowing interstitial fluid. ${ }^{38}$

\subsection{In vitro osteogenic potential of the new bone graft}

To evaluate the osteogenic differentiation ability of the new bone graft, Alizarin Red S and alkaline phosphatase (ALP) dyes were subsequently detected in the experimental and control groups. Regarding the ALP staining, the blue area highlights the better osteogenic differentiation of hMSCs. Fig. 10A and B shows more blue area in the experimental group than that in the control group, which means a better osteogenic differentiation in the experimental group. Alizarin Red S staining could also indicate osteogenic differentiation. Fig. 10C and D show more red nodules present in the experimental group compared with the control group. Along with hMSCs differentiating to

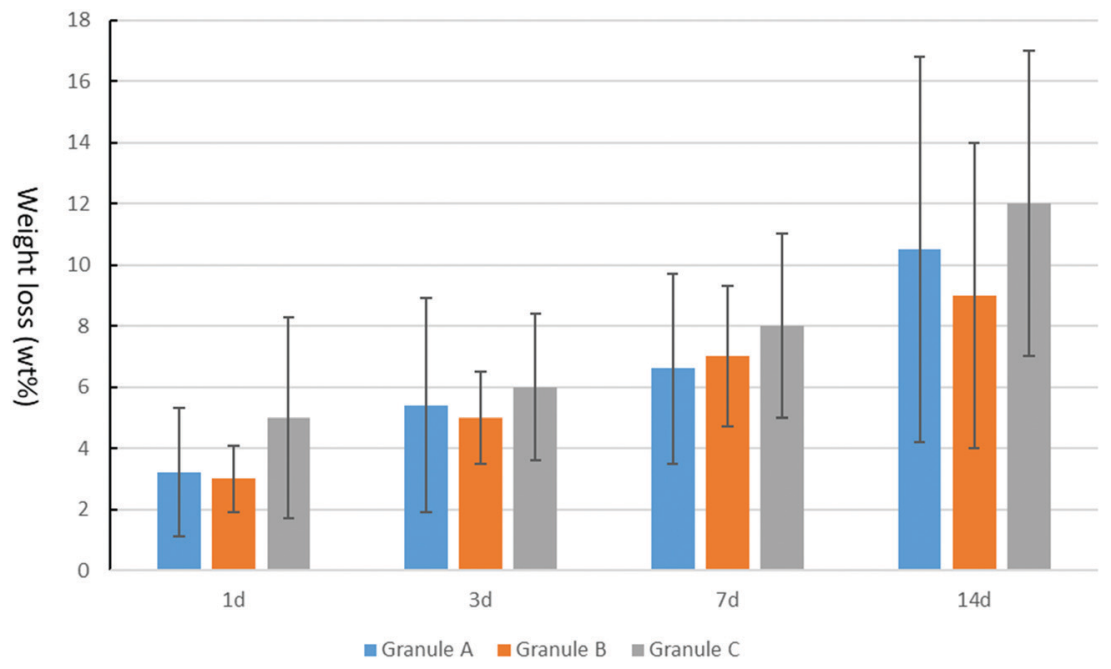

Fig. 8 In vitro degradation rate (wt\%) of granules $A, B$ and $C$ after $1,3,7$ and 14 days. 


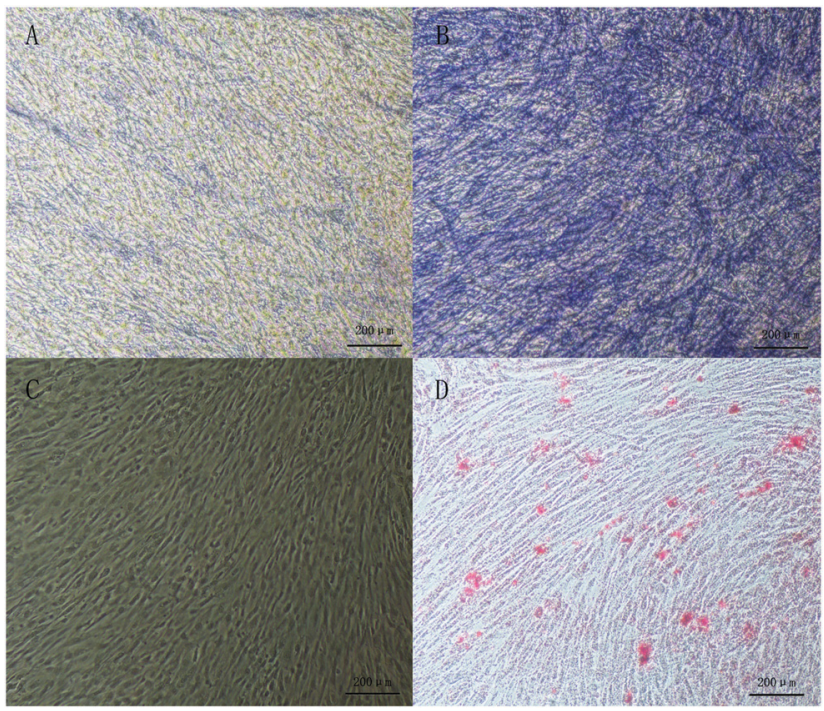

Fig. 10 Alkaline phosphatase (ALP) activity of human mesenchymal stem cells (hMSCs) cultured on (A) a tissue culture plate and (B) granule $A$ Calcium nodules stained by Alizarin Red on (C) a tissue culture plate and (D) granule A. Cells were cultured for 14 days.

osteoblasts, $\mathrm{Ca}^{2+}$ will be secreted around the cells creating calcium nodules, which could be dyed into a red spot by the Alizarin Red solution. In previous studies, osteoblast cellular response especially viability and proliferation could be enhanced in TCP and HA scaffolds compared with pure TCP scaffolds. ${ }^{39}$ However, one study focused on the improvement of bone regeneration capability of ceramic scaffolds and demonstrated that accelerated release of calcium ions could induce seeded MSCs to differentiate into osteoblast-like cells without osteogenic media, as determined by expression of osteogenic markers in vitro. ${ }^{40}$ Plamer et al. reported that pyrophosphate could exert a physiological effect, stimulating osteoblast differentiation markers and extracellular matrix gene expression. ${ }^{20}$ Therefore, osteogenic potential of the multi-phased injectable bone graft could be expected.

\subsection{Bone formation in the specific animal model}

Micro-CT images (Fig. 11) show the bone formation after one and two months implantation for both the control and testing groups. No obvious new bone formation was observed in one and two months for the control group. As a comparison, new cancellous bone can be observed in the testing group, especially after two months implantation. In the first month, we can still see the injected granules, but there are only a few after two months. Fig. 12 shows the bone volume in the femoral head defect after implantation. The experimental group has a much higher bone volume than the control group at both one and two months $(p<0.05)$. For biomaterials applied in healing femoral head disease, Zhu et al. implanted 3D-printed porous titanium in a rabbit femoral head defeat, which exhibited a better regeneration and supporting effect. ${ }^{41}$ However, it lacks the ability to degrade and is hard to take out again, which would cause difficulties in secondary treatment in femoral head

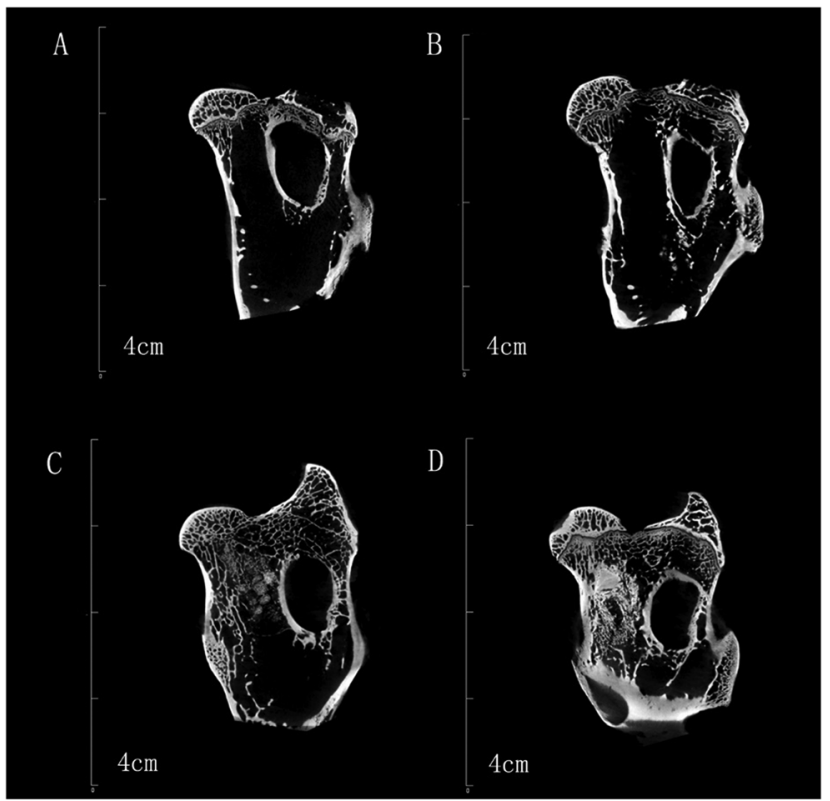

Fig. 11 Micro-CT analysis: control group after (A) 1 and (B) 2 months; experimental group after (C) 1 and (D) 2 months.

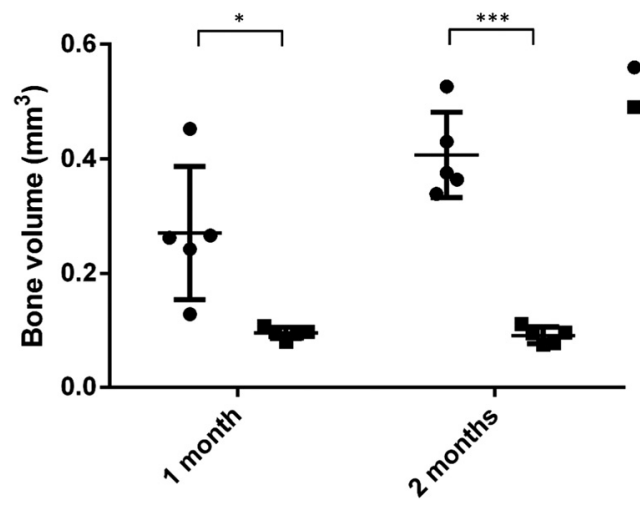

Fig. 12 Bone volume in the femoral head defect 1 and 2 months post operation ( ${ }^{\star} P<0.05 ;{ }^{*} P \leq 0.001$; two-tailed $T$-test). Experimental group: using granule $A$; control group: without using materials.

disease patients such as fracture and arthroplasty if applied in clinics in the future. The injectable particles in our study not only achieved a better effect in femoral head defect regeneration but were also minimally invasive and could reduce the chance of secondary surgery if used in clinics. Civinini $e t$ al. tried to inject a calcium sulphate/calcium phosphate composite into femoral head necrosis patients and finally found that it could relieve hip pain and prevent the progression of $\mathrm{ONFH}$ in the majority of the patients compared with injection of autologous bone marrow. ${ }^{42}$ Chang et al. combined poly(propylene fumarate) (PPF) and calcium phosphate cement (CPC) and studied their effect in femoral head repair, and they found that it could provide appropriate mechanical strength after femoral head core-decompression and provide the property of osteoconductivity.$^{43}$ Many papers have reported that biphasic calcium phosphate (BCP) ceramics, i.e. HA/ $\beta$-TCP, were better than one 


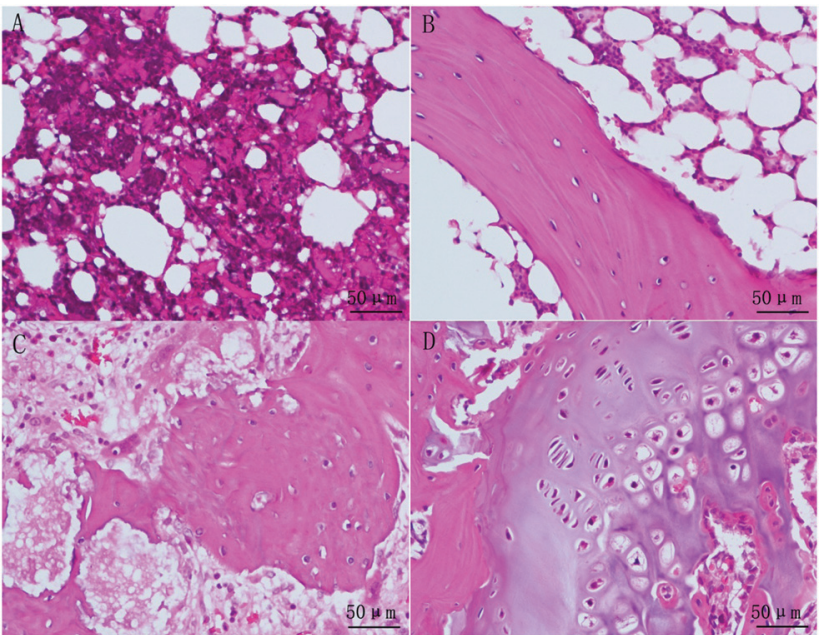

Fig. 13 Histological sections of rabbit femoral head after implantation staining using hematoxylin and eosin ( $\mathrm{HE}$ ). (A and B) One and two months for the control group; ( $C$ and $D)$ one and two months for the experimental group.

phase calcium phosphate, i.e. HA, ACP and $\beta$-TCP, and have proven efficacy in clinical testing. ${ }^{44-49}$ The ratio between HA and $\beta$-TCP did influence the biological and clinical results. ${ }^{48,49}$ However, no publication, as far as we know, reported an optimized biological effect of 3 phased (HA $/ \alpha-\mathrm{TCP} / \beta$-TCP) bioceramics. In this study, we chose the composition of granule A based on its structural properties and degradation rate. The pores in bone cement after setting are generally small, less than $10 \mu \mathrm{m}$, which is not large enough for new bone formation. Injecting a granule-like bone graft could form a macroporous scaffold in situ. A large pore structure (larger than $100 \mu \mathrm{m}$ ) could enhance new bone formation.

HE staining (Fig. 13) shows that most of the area was fat tissue and inflammation in the one month section of the control group (Fig. 13A). The 2 month results of the section of the control group (Fig. 13B) showed a bit of woven bone formation but most of the tissue was still fat. A small portion of new woven bone tissue and some connective tissue were observed in the 1 month section of the experimental group (Fig. 13C). Mature bone tissue such as lamellar bone can be observed on the 2 month section (Fig. 13D). It was found that the amount of new bone tissue in the experimental group was obviously more than that of the control group, and the maturity of the bone tissue was higher than that of the control group. Sirius red staining (Fig. 14) was performed to observe collagen production, which can predict the bone regeneration process with more type I collagen expression. In the staining, the yellow or red fibre represents type I collagen. The loose colourful network (Fig. 14A) represents type II collagen. The green fibre is type III collagen, and the light yellow fibre is type IV collagen. Most of the bone tissue contained type I collagen, showing a yellow or red strip-like distribution. A large amount of type I collagen was distributed in the section of the experimental group, which is related to bone tissue, while in the control group, there was less type I collagen. In summary, both HE and

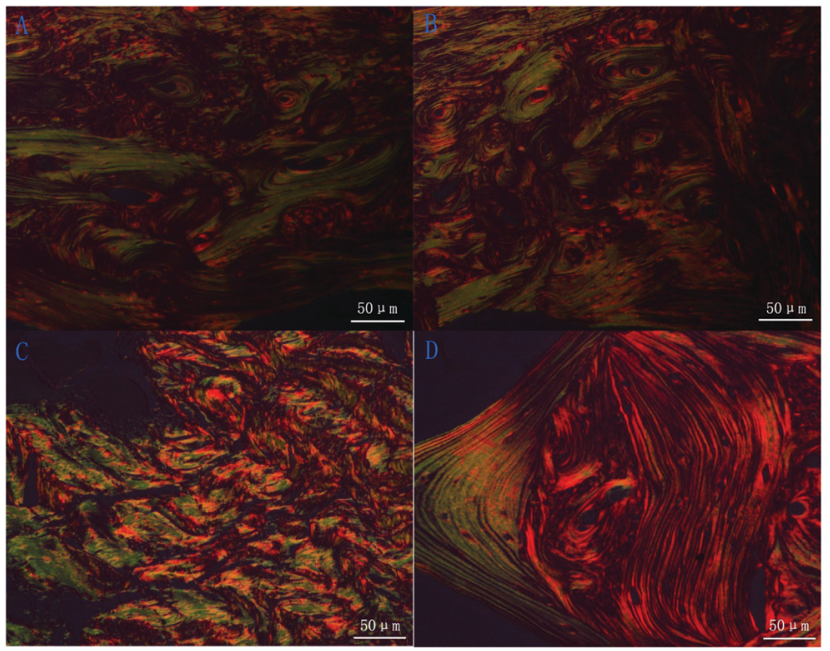

Fig. 14 Histological sections of rabbit femoral head after implantation staining using Sirius Red. (A and B) One and two months for the control group; ( $C$ and $D$ ) one and two months for the experimental group.

SR staining proved that significant new bone formation occurred in the experimental group.

\section{Conclusions}

An easily cemented multi-phased calcium phosphate bone graft has been fabricated for the treatment of femoral head necrosis. The phase composition, pore size and porosity, and degradation of the grafts could be adjusted via controlling the setting process and the size of the PEG particles. Drying and autoclaving can also affect the final phase composition. Granule A, obtained using PEG 100-600 $\mu \mathrm{m}$, was chosen for the in vitro and in vivo studies. The granules could stimulate cell proliferation with increased time. The granules can be injected into a femoral head defect in rabbits. New cancellous bone could be clearly observed even after one month of implantation. HE staining indicated that mature bone tissue was formed after two months of implantation, and SR staining showed collagen formation, which is related to new bone formation. The results confirmed an important application of this injectable multi-phased $\mathrm{CaP}$ granule for the treatment of femoral head necrosis.

\section{Conflicts of interest}

The authors declare that they have no conflicts of interest.

\section{Acknowledgements}

This work was supported by the Beijing Natural Science Foundation youth project (7184325), the China Postdoctoral Foundation No. 62 general program, and The Swedish Foundation for International Cooperation in Research and Higher Education (STINT, CH2015-6394). 


\section{References}

1 H. J. Mankin, N. Engl. J. Med., 1992, 326, 1473-1479.

2 M. A. Mont and D. S. Hungerford, J. Bone Jt. Surg., Am. Vol., 1995, 77, 459-474.

3 E. M. Camporesi, G. Vezzani, G. Bosco, D. Mangar and T. L. Bernasek, J. Arthroplasty, 2010, 25, 118-123.

4 D. Arbab and D. P. Konig, Dtsch. Arztebl. Int., 2016, 113, 31-38.

5 S. K. Tripathy, T. Goyal and R. K. Sen, Indian J. Orthop., 2015, 49, 28-45.

6 Y. Fillingham and J. Jacobs, Bone Jt. J., 2016, 98-B, 6-9.

7 T. Ghassemi, A. Shahroodi, M. H. Ebrahimzadeh, A. Mousavian, J. Movaffagh and A. Moradi, Arch. Bone Jt. Surg., 2018, 6, 90-99.

8 J. M. Bouler, P. Pilet, O. Gauthier and E. Verron, Acta Biomater., 2017, 53, 1-12.

9 T. Kasuga, Acta Biomater., 2005, 1, 55-64.

10 M. Bohner, Biomaterials, 2009, 30, 6403-6406.

11 R. G. Carrodeguas and S. De Aza, Acta Biomater., 2011, 7, 3536-3546.

12 M. Vallet-Regí and J. M. González-Calbet, Prog. Solid State Chem., 2004, 32, 1-31.

13 E. Boanini, M. Gazzano and A. Bigi, Acta Biomater., 2010, 6, 1882-1894.

14 S. V. Dorozhkin, Biomaterials, 2010, 31, 1465-1485.

15 S. V. Dorozhkin, Materials, 2009, 2, 399.

16 S. V. Dorozhkin, Acta Biomater., 2012, 8, 963-977.

17 W. R. Walsh, F. Vizesi, D. Michael, J. Auld, A. Langdown, R. Oliver, Y. Yu, H. Irie and W. Bruce, Biomaterials, 2008, 29, 266-271.

18 J. Zhang, X. Luo, D. Barbieri, A. M. Barradas, J. D. de Bruijn, C. A. Van Blitterswijk and H. Yuan, Acta Biomater., 2014, 10, 3254-3263.

19 A. Gatti, D. Zaffe and G. Poli, Biomaterials, 1990, 11, 513-517.

20 M. Pujari-Palmer, S. Pujari-Palmer, X. Lu, T. Lind, H. Melhus, T. Engstrand, M. Karlsson-Ott and H. Engqvist, PLoS One, 2016, 11, e0163530.

21 Y. Murakami, Y. Honda, T. Anada, H. Shimauchi and O. Suzuki, Acta Biomater., 2010, 6, 1542-1548.

22 A. John, S. Abiraman, H. Varma, T. Kumari and P. Umashankar, Bull. Mater. Sci., 2002, 25, 141-154.

23 M.-H. Hong, S.-M. Kim, M.-H. Han, Y. H. Kim, Y.-K. Lee and D. S. Oh, Ceram. Int., 2014, 40, 6095-6102.

24 P. Habibovic, T. M. Sees, M. A. van den Doel, C. A. van Blitterswijk and K. de Groot, J. Biomed. Mater. Res., Part A, 2006, 77, 747-762.

25 A. Barba, A. Diez-Escudero, Y. Maazouz, K. Rappe, M. Espanol, E. B. Montufar, M. Bonany, J. M. Sadowska, J. Guillem-Marti and C. Öhman-Mägi, ACS Appl. Mater. Interfaces, 2017, 9, 41722-41736.

26 V. Karageorgiou and D. Kaplan, Biomaterials, 2005, 26, 5474-5491.
27 P. Kasten, I. Beyen, P. Niemeyer, R. Luginbuhl, M. Bohner and W. Richter, Acta Biomater., 2008, 4, 1904-1915.

28 M. P. Ginebra, E. Fernández, F. C. M. Driessens, M. G. Boltong, J. Muntasell, J. Font and J. A. Planell, J. Mater. Sci.: Mater. Med., 1995, 6, 857-860.

29 S. Y. Kim and S. H. Jeon, J. Ind. Eng. Chem., 2012, 18, 128-136.

30 S. V. Dorozhkin, Materials, 2009, 2, 221.

31 H. H. K. Xu, P. Wang, L. Wang, C. Bao, Q. Chen, M. D. Weir, L. C. Chow, L. Zhao, X. Zhou and M. A. Reynolds, Bone Res., 2017, 5, 17056.

32 A. J. Ambard and L. Mueninghoff, J. Prosthodontics, 2006, 15, 321-328.

33 H.-a. Kodama, Y. Amagai, H. Sudo, S. Kasai and S. Yamamoto, Jpn. J. Oral Biol., 1981, 23, 899-901.

34 Y. Gao, W.-L. Cao, X.-Y. Wang, Y.-D. Gong, J.-M. Tian, N.-M. Zhao and X.-F. Zhang, J. Mater. Sci.: Mater. Med., 2006, 17, 815-823.

35 J. Zhang, F. Tancret and J.-M. Bouler, Mater. Sci. Eng., C, 2011, 31, 740-747.

36 J.-C. Chen, C.-L. Ko, C.-J. Shih, Y.-C. Tien and W.-C. Chen, J. Dent., 2012, 40, 114-122.

37 R. Olkowski, P. Kaszczewski, J. Czechowska, D. Siek, D. Pijocha, A. Zima, A. Ślósarczyk and M. LewandowskaSzumieł, J. Mater. Sci.: Mater. Med., 2015, 26, 270.

38 L. E. Rustom, T. Boudou, S. Lou, I. Pignot-Paintrand, B. W. Nemke, Y. Lu, M. D. Markel, C. Picart and A. J. W. Johnson, Acta Biomater., 2016, 44, 144-154.

39 M. Castilho, C. Moseke, A. Ewald, U. Gbureck, J. Groll, I. Pires, J. Teßmar and E. Vorndran, Biofabrication, 2014, 6, 015006.

40 Y.-J. Seol, J. Y. Park, J. W. Jung, J. Jang, R. Girdhari, S. W. Kim and D.-W. Cho, Tissue Eng., Part A, 2014, 20, 2840-2849.

41 W. Zhu, Y. Zhao, Q. Ma, Y. Wang, Z. Wu and X. Weng, J. Mater. Sci.: Mater. Med., 2017, 28, 62.

42 R. Civinini, P. De Biase, C. Carulli, F. Matassi, L. Nistri, R. Capanna and M. Innocenti, Int. Orthop., 2012, 36, 1583-1588.

43 C.-H. Chang, T.-C. Liao, Y.-M. Hsu, H.-W. Fang, C.-C. Chen and F.-H. Lin, Biomaterials, 2010, 31, 4048-4055.

44 R. F. Ellinger, E. B. Nery and K. L. Lynch, Int. J. Periodontics Restorative Dent., 1986, 6, 22-33.

45 C. Lindgren, A. Mordenfeld, C. B. Johansson and M. Hallman, Int. J. Oral Maxillofac. Implants, 2012, 27, 1151-1162.

46 F. Monchau, A. Lefevre, M. Descamps, A. Belquin-myrdycz, P. Laffargue and H. F. Hildebrand, Biomol. Eng., 2002, 19, 143-152.

47 S. Yamada, D. Heymann, J. M. Bouler and G. Daculsi, Biomaterials, 1997, 18, 1037-1041.

48 S. V. Dorozhkin, Ceram. Int., 2016, 42, 6529-6554.

49 J. M. Bouler, P. Pilet, O. Gauthier and E. Verron, Acta Biomater., 2017, 53, 1-12. 